\title{
Combined use of steady-state fluorescence emission and anisotropy of merocyanine 540 to distinguish crystalline, gel, ripple, and liquid crystalline phases in dipalmitoylphosphatidylcholine bilayers
}

\author{
Hannabeth A Franchino ${ }^{1}$, Brett C Johnson ${ }^{1}$, Steven K Neeley ${ }^{1}$, Rajeev B Tajhya ${ }^{1}$, Mai P Vu', \\ Heather A Wilson-Ashworth², John D Bell ${ }^{1 *}$
}

\footnotetext{
* Correspondence: john_bell@byu. edu

${ }^{1}$ Department of Physiology and Developmental Biology, Brigham Young University, Provo, Utah 84602, USA.
}

\begin{abstract}
The various lamellar phases of dipalmitoylphosphadtidylcholine bilayers with and without cholesterol were used to assess the versatility of the fluorescent probe merocyanine 540 through simultaneous measurements of emission intensity, spectral shape, and steady-state anisotropy. Induction of the crystalline phase $\left(L_{c}{ }^{\prime}\right)$ by preincubation at $4^{\circ} \mathrm{C}$ produced a wavelength dependence of anisotropy which was strong at 15 and $25^{\circ} \mathrm{C}$, weak at $38^{\circ} \mathrm{C}$, and minimal above the main transition $\left(>\sim 41.5^{\circ} \mathrm{C}\right)$ or after returning the temperature from 46 to $25^{\circ} \mathrm{C}$. The profile of anisotropy values across this temperature range revealed the ability of the probe to detect crystalline, gel $\left(L_{\beta}{ }^{\prime}\right)$, and liquid crystalline $\left(L_{\alpha}\right)$ phases. The temperature dependence of fluorescence intensity was additionally able to distinguish between the ripple $\left(P_{\beta}{ }^{\prime}\right)$ and gel phases. In contrast, the shape of the emission spectrum, quantified as the ratio of merocyanine monomer and dimer peaks (585 and $621 \mathrm{~nm}$ ), was primarily sensitive to the crystalline and gel phases because dimer fluorescence requires a highly-ordered environment. This requirement also explained the diminution of anisotropy wavelength dependence above $25^{\circ} \mathrm{C}$. Repetition of experiments with vesicles containing cholesterol allowed creation of a phase map. Superimposition of data from the three simultaneous measurements provided details about the various phase regions in the map not discernible from any one of the three alone. The results were applied to assessment of calcium-induced membrane changes in living cells.

PACS Codes: 87.16.dt.
\end{abstract}

\section{Introduction}

Fluorescence spectroscopy is a useful biological technique for studying membrane structure that can be applied directly to living cells. Measurements in real time with living cells, before and after treatments with pharmacological agents, are most easily accomplished using steady-state measurements. If one could increase the amount of biophysical information available from those measurements, it would reduce the need for dual labeling or comparisons of parallel experiments with different probes. For example, it has been found with the probe laurdan that measurement of both steady-

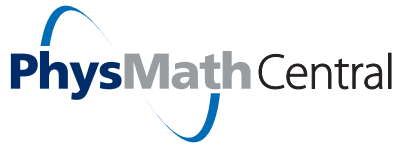

c 2009 Franchino et al This is an Open Access article distributed under the terms of the Creative Commons Attribution License (http:// creativecommons.org/licenses/by/2.0), which permits unrestricted use, distribution, and reproduction in any medium, provided the original work is properly cited. 
state anisotropy and emission spectrum shape can yield more detailed information about membrane phase properties in both artificial and natural membranes than either measurement alone [1,2]. Merocyanine 540 (MC540) seems like an ideal candidate for a similar approach but with greater resolution and flexibility. Its emission intensity is environment-sensitive, which allows it to be used in flow cytometry experiments to quantify cell subpopulations with differing biophysical membrane properties [3,4]. It binds to the plasma membrane of most cells at low concentrations (i.e. Ref. [3-8]). Furthermore, it has been used extensively for studies of membrane properties during apoptosis (i.e. Ref. [3,4,8-12]). Because of the sensitivity of MC540 to lipid-packing density and the ability of the probe to partition into different membrane locations depending on lipid phase [7,13-17], we postulated that it might be able to provide detailed information about membrane dynamics through multiple simultaneous steadystate measurements. We have used bilayers made of dipalmitoylphosphatidylcholine (DPPC) with and without cholesterol to assess the extent to which such might be the case.

The characteristics of pure DPPC in its four lamellar phases have been well-studied with biophysical methods such as differential scanning calorimetry, $\mathrm{x}$-ray diffraction, and various spectroscopic techniques [18-23]. These include solid-ordered phases-crystalline $\left(\mathrm{L}_{\mathrm{c}}{ }^{\prime}\right)$, gel $\left(\mathrm{L}_{\beta}{ }^{\prime}\right)$, and ripple $\left(\mathrm{P}_{\beta}{ }^{\prime}\right)$-and the liquid-disordered or liquid crystalline phase $\left(L_{\alpha}\right)$. More recently, liquid-ordered $\left(L_{o}\right)$ phases have been observed in the presence of cholesterol [24-30]. Additionally, at specific cholesterol concentrations, the $\mathrm{L}_{\alpha}$ and $\mathrm{L}_{\mathrm{o}}$ phases become more complex due to formation of extended superlattice structures [31-33].

The $\mathrm{L}_{\mathrm{c}}$ ' phase of pure DPPC is found at low temperatures and can be induced in vesicles when stored at $-5^{\circ} \mathrm{C}$ for 2 hours or longer or at $4^{\circ} \mathrm{C}$ for more than 24 hours $[18,34]$. In this phase, both the hydrocarbon chains of the lipids as well as the hydrophilic heads are tightly packed with highly restricted motion [35]. An increase in temperature causes the lipids to undergo the sub-transition at $\sim 18^{\circ} \mathrm{C}$ and enter the $\mathrm{L}_{\beta}$ ' phase, which is characterized by increases in the spatial area occupied by the phospholipids, rotational freedom of movement, and hydration and tilt of the heads away from the bilayer normal [35].

The transition between the $\mathrm{L}_{\beta}$ ' and $\mathrm{P}_{\beta}$ ' phases of pure DPPC is classified as the pretransition (centered at about $33-34^{\circ} \mathrm{C}$ ) and is usually detected with differential scanning calorimetry $[20,21]$. The $P_{\beta}$ ' phase is characterized by an increase in the interfacial area of the membrane as the lipid polar head groups occupy greater space [35]. In the case of multilamellar vesicles, the surface morphology changes at this point from being planar to instead bending into a series of periodic ripples [35].

Merocyanine 540 has a negatively-charged sulfate group that creates a permanent dipole moment which affects the binding locations of the probe and its tendency to exist as either monomers or dimers in the membrane [36]. The monomeric form of MC540 resides near the lipid head groups at an orientation parallel to the phospholipid chains [16]. Alternatively, MC540 can form an anti-parallel "sandwich" dimer [17]. It has been proposed that this dimer occupies a different region of the membrane compared to the monomer, although the exact orientation of the probe and whether that region is deep or superficial in the membrane are uncertain [16,37]. Emission intensity spectra show that the monomer fluoresces at a shorter wavelength than the 
dimer (585 nm and $621 \mathrm{~nm}$, respectively) [17,37]. While the overall dimer fluorescence intensity is lower than that of monomers, dimers do contribute a noticeable amount to the emission intensity of MC540 when they reside in a highly packed environment such as exists in the membrane below the main phase transition [17]. Dimers are known to be non-fluorescent in both aqueous environments and when occupying membranes in the liquid-crystalline phase [17]. The introduction of cholesterol to the membrane is also reported to decrease the fluorescence of the dimer [15].

In this study, we have examined the versatility of using simultaneous measurements of MC540 anisotropy, emission intensity, and spectral shape to resolve the various DPPC lamellar phases. These experiments were then applied to two-component bilayers with multiple mole fractions of cholesterol. Finally, the study was complemented with a brief proof-of-concept application to living cells.

\section{Methods}

\section{Formation of vesicles}

Vesicles were made by combining $1 \mu$ mole DPPC (Avanti Polar Lipids, Birmingham, AL) dissolved in chloroform with 5-6.8 nmoles MC540 to create membranes with a 1:145-1:200 probe-to-lipid ratio. Control experiments at a variety of ratios demonstrated that MC540 caused minimal perturbation to the membrane structure at ratios less than 1:100. Samples were dried under $\mathrm{N}_{2}$ gas and re-suspended in $1 \mathrm{ml}$ of $\mathrm{pH} 7$ citrate buffer ( $20 \mathrm{mM}$ sodium citrate/citric acid, $150 \mathrm{mM} \mathrm{KCl}$ ). The co-dispersing of MC540 with lipids during vesicle preparation was done to ensure that the probe would be properly equilibrated with the bilayers. For some experiments, DPPC was mixed with cholesterol (both dissolved in chloroform) at various mole ratios $(1 \mu$ mole total lipid) prior to drying and suspension in citrate buffer. Suspensions were then incubated in a shaking water bath at $\geq 50^{\circ} \mathrm{C}$ for 10 -min intervals and vortexed between each incubation (total incubation time was one hour). The vesicles were stored in a covered container either at room temperature or at $4^{\circ} \mathrm{C}$ for at least $24 \mathrm{~h}$ before use. Samples containing cholesterol were kept refrigerated and used soon after preparation to minimize oxidation [38].

\section{Steady-state fluorescence measurements}

Steady-state fluorescence was assayed using a Fluoromax-3 (Horiba Jobin Yvon, Edison, NJ) or PC-1 (ISS, Champaign, IL) photon-counting spectrofluorometer. Temperature was controlled using a circulating water bath, and sample homogeneity was maintained by magnetic stirring. Samples were equilibrated in the fluorometer sample compartment for $15 \mathrm{~min}$ at the initial experimental temperature. Following that initial equilibration, fluorescence data were acquired. The sample was then given $10 \mathrm{~min}$ for reequilibration when temperature was adjusted by single-degree increments or $15 \mathrm{~min}$ when the adjustment was more than $5^{\circ} \mathrm{C}$. The probe was excited at $540 \mathrm{~nm}$. Emission intensity spectra were measured between 550 and $700 \mathrm{~nm}$. Data were reported either as total intensity (calculated at $585 \mathrm{~nm}$ ) or as the peak ratio (intensity at $585 \mathrm{~nm}$ divided by that at $621 \mathrm{~nm}$ ). Anisotropy was assayed at $5 \mathrm{~nm}$ wavelength increments between 580 and $625 \mathrm{~nm}$ using Glan-Thompson polarizers and standard techniques [39]. Interference from scattered light was estimated from vesicle samples without probe and found to be negligible at the wavelengths and conditions used. Spectra 
shown in the figures are therefore uncorrected for light scatter. Excited-state lifetime values for interpretation of anisotropy results were obtained using an ISS Koala spectrofluorometer in the frequency-modulation domain at the Laboratory for Fluorescence Dynamics (Irvine, CA). A bandpass filter with cutoff at $585 \mathrm{~nm}$ and a longpass filter (50\% transmission at $600 \mathrm{~nm}$ ) were used to isolate the blue and red edges of MC540 emission.

\section{Color phase maps}

Maps of membrane phase properties in two dimensions (temperature and cholesterol concentration) were generated from anisotropy, emission intensity, and peak ratio measurements as described previously [40]. Data were normalized and scaled to the value observed at $24-25^{\circ} \mathrm{C}$ and the extreme value observed at temperatures above $45^{\circ} \mathrm{C}$. The normalization was justified based on regression of the raw values of each parameter at these temperature extremes. In each case, the statistical analysis revealed that differences in parameter values at the ends of the temperature profile were due to random rather than systematic effects. Each normalized datum was assigned a color value between 0 and 255 for its respective hue (anisotropy = green, intensity = red, ratio = blue). For intensity and ratio, a normalized value of one was assigned a color value of 255 (brightest) and values less than one were multiplied by 255 to convert them to scaled color intensities. For anisotropy, color values were assigned in a similar fashion with one exception. After initial scaling, the resulting color values were then subtracted from 255 so that color brightness of the green color map followed the same pattern as those for the other two parameters (i.e. brighter color = more fluid membrane).

\section{Cultured Cells}

S49 murine lymphoma cells were cultured and prepared for experiments as described [41]. Two $\mathrm{ml}$ of washed cells were added to a fluorometer sample cell with $170 \mathrm{nM}$ MC540 and allowed to incubate at $37^{\circ} \mathrm{C}$ for $5 \mathrm{~min}$. Anisotropy was assayed at $5 \mathrm{~nm}$ wavelength increments between 580 and $625 \mathrm{~nm}$. (Emission intensity and peak ratio were also calculated from those data.) Following anisotropy measurements, a calcium ionophore, ionomycin, was added (300 $\mathrm{nM})$ and allowed to incubate with the sample for $10 \mathrm{~min}$. Anisotropy data were then collected again.

\section{Results}

\section{Steady state anisotropy}

In order to achieve the crystalline $\left(\mathrm{L}_{\mathrm{c}}{ }^{\prime}\right)$ phase, vesicles were incubated at $4{ }^{\circ} \mathrm{C}$ for at least $24 \mathrm{~h}$. As shown in Figure 1A, MC540 anisotropy values for vesicles in the $\mathrm{L}_{\mathrm{c}}$ ' phase $\left(15^{\circ} \mathrm{C}\right.$, green lines and symbols) depended strongly on emission wavelength. The decreasing trend in anisotropy between $580 \mathrm{~nm}$ and $625 \mathrm{~nm}$ was statistically significant based on linear regression (slope $95 \%$ confidence interval $=-0.004$ to $-0.003 \mathrm{~nm}^{-1}$, $\left.p<0.0001, \mathrm{r}^{2}=0.90\right)$. The anisotropy measurements were repeated at $25^{\circ} \mathrm{C}$ (black), a temperature above the sub-transition $\left(\sim 18^{\circ} \mathrm{C}\right.$, Ref. [18]) from the $\mathrm{L}_{\mathrm{c}}$ ' to the $\mathrm{L}_{\beta}$ ' (gel) phase. When this was done, the wavelength dependence persisted, although the overall anisotropy values were lower compared to those at $15^{\circ} \mathrm{C}$ (slope $95 \%$ confidence interval $=-0.005$ to $-0.004 \mathrm{~nm}^{-1}, p<0.0001, \mathrm{r}^{2}=0.91$; effects of temperature and wavelength were both significant by two-way analysis of variance $(p<0.0001)$ with significant 


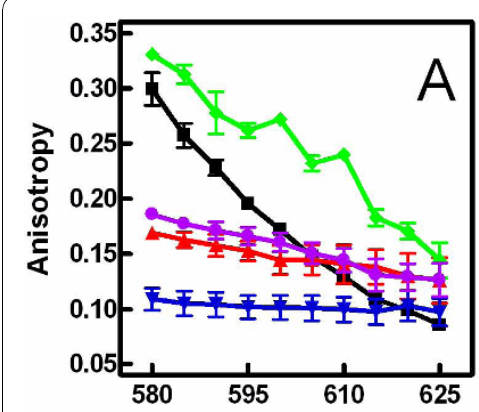

Figure 1 MC540 steady-state anisotropy spectra at various temperatures. Panel A: Vesicles were stored at $4^{\circ} \mathrm{C}$. Anisotropy was assayed at four temperatures 15 (green), 25 (black), 38 (red), and $46^{\circ} \mathrm{C}$ (blue). Following collection of anisotropy data at $46^{\circ} \mathrm{C}$, the samples were cooled back to $25^{\circ} \mathrm{C}$ and anisotropy was re-measured (violet). Error bars represent the SEM $(n=3-7)$ vesicle samples. Panel B: Vesicles were stored at room temperature and anisotropy was assayed at 25 (black), 38 (red), and $46^{\circ} \mathrm{C}$ (blue). Panel C: The vesicles of Panel B were stored overnight at $4^{\circ} \mathrm{C}$ after the data in Panel B had been gathered. The anisotropy was then assayed again at 25 (black), 38 (red), and $46^{\circ} \mathrm{C}$ (blue). Error bars represent the range of two samples in panels $\mathrm{B}$ and $\mathrm{C}$. The data at $15^{\circ} \mathrm{C}$ from Panel $\mathrm{A}$ were included in Panels $\mathrm{B}$ and $\mathrm{C}$ to facilitate comparisons.

interaction ( $p=0.001)$ that only accounts for $2 \%$ of the variance; the temperature effect accounted for $19 \%$ of the variance). However, once the sample passed through the pre-transition from the $\mathrm{L}_{\beta}$ ' to the $\mathrm{P}_{\beta}$ ' phase $\left(\sim 30^{\circ} \mathrm{C},[20]\right)$, changes across the wavelength spectrum were significantly reduced $\left(38^{\circ} \mathrm{C}\right.$, red) and were absent above the main phase transition $\left(46^{\circ} \mathrm{C}\right.$, blue; $\left.\mathrm{t}_{\mathrm{m}}=\sim 41.5^{\circ} \mathrm{C},[22,23]\right)$. The slope $95 \%$ confidence interval at $38^{\circ} \mathrm{C}=-0.0015$ to $-0.0004 \mathrm{~nm}^{-1}, p=0.002, \mathrm{r}^{2}=0.30$; at $46^{\circ} \mathrm{C}=-0.0007$ to $0.0003 \mathrm{~nm}^{-1}, p=0.4, \mathrm{r}^{2}=0.01$.

One of the well known characteristics of the $\mathrm{L}_{\mathrm{c}}$ ' phase is that the kinetics of the transition to and from it are slow (time scale of hours to days, depending on the experimental conditions) such that significant hysteresis is observed as one passes through the sub-transition $[19,34,42,43]$. We used this phenomenon to determine whether the wavelength dependence at low temperature was due to the $\mathrm{L}_{\mathrm{c}}$ ' phase by returning the temperature to $25^{\circ} \mathrm{C}$ after the vesicles had passed through the main transition. As illustrated by the violet line and symbols in Figure $1 \mathrm{~A}$, the wavelength dependence at $25^{\circ}$ $\mathrm{C}$ following a temperature reversal was greatly attenuated and now indistinguishable from the observations at $38^{\circ} \mathrm{C}$ (slope $95 \%$ confidence interval $=-0.002$ to $-0.001 \mathrm{~nm}^{-1}$, $\left.p<0.0001, \mathrm{r}^{2}=0.55\right)$. This result indicates that the vesicles were affected by their recent thermal history and suggests that the original data at $25^{\circ} \mathrm{C}$ represented vestigial effects of the $\mathrm{L}_{\mathrm{c}}$ ' phase because of hysteresis through the sub-transition. This interpretation was confirmed by experiments with vesicles that were stored at room temperature continuously since their manufacture (Figure 1B). Similar to the data for vesicles returned to $25^{\circ} \mathrm{C}$ after passing through high temperature (violet symbols in Figure 1A), the anisotropy trend at $25^{\circ} \mathrm{C}$ mirrored the trend at $38^{\circ} \mathrm{C}$ in this experiment. Furthermore, vesicles stored at room temperature and displaying behavior like Figure $1 \mathrm{~B}$ converted to the pattern of Figure $1 \mathrm{~A}$ after they were subsequently placed at $4^{\circ} \mathrm{C}$ for at least $24 \mathrm{~h}$ (Figure $1 \mathrm{C}$ ). This control experiment verified that the results of Figure 1 were reflective of the recent thermal history of the bilayers rather than an artifact of incomplete equilibration during vesicle manufacture. 
Figure 2 displays a detailed temperature profile of MC540 anisotropy for vesicles stored previously at $4^{\circ} \mathrm{C}$ ("refrigerated"). The hysteretic recovery of anisotropy from the $\mathrm{L}_{\mathrm{c}}$ ' phase was evident for the data from 20 to $32^{\circ} \mathrm{C}$. The triangle and dotted line indicate the anisotropy level upon return of the vesicles from high temperature. The main phase transition to the $\mathrm{L}_{\alpha}$ phase was also apparent in the data at $40-42^{\circ} \mathrm{C}$.

\section{Emission intensity}

Evidence for all four lamellar phases was observed by comparing the temperature profile of emission intensity between refrigerated (Figure 3, solid circles) and equilibrated vesicles (heated to $50^{\circ} \mathrm{C}$ prior to measurements, open circles). The difference in intensity between 25 and $35^{\circ} \mathrm{C}$ in these equilibrated vesicles presumably represented the $\mathrm{L}_{\beta}$ ' and $\mathrm{P}_{\beta}$ ' phases based on their corresponding temperature ranges [21]. Analogous to the explanation of Figure 2, the additional reduction in intensity observed at low temperatures in the refrigerated vesicles (solid circles, below $29^{\circ} \mathrm{C}$ ) appeared to reflect vestigial effects of the $\mathrm{L}_{\mathrm{c}}$ ' phase. The highest intensity in both vesicle preparations corresponded to the $\mathrm{L}_{\alpha}$ phase.

\section{Emission spectrum shape}

As shown in Figure 4A, the emission spectrum shape differed between high and low temperatures. The low-temperature spectrum consisted of two peaks (see inset), reflecting separate populations of MC540 in fluorescent monomer $(585 \mathrm{~nm})$ and dimer $(621 \mathrm{~nm})$ configurations [37]. The single emission peak observed at higher temperatures (dashed and heavy solid curves) presumably reflected monomer fluorescence since prior research has shown that the dimer subpopulation no longer fluoresces at temperatures above the main phase transition [17]. The large increase in monomer fluorescence intensity has been interpreted to represent enhanced probe binding to the membrane and perhaps loss of optical interference and quenching from dimers $[13,14,17,44]$. Previously, the spectrum showing both monomers and dimers was attributed to the gel phase $\left(\mathrm{L}_{\beta}{ }^{\prime}\right)$ of the lipids [17]. However, the emission spectrum at $25^{\circ} \mathrm{C}$ from vesicles previously equilibrated at high temperature mostly lacked the dimer

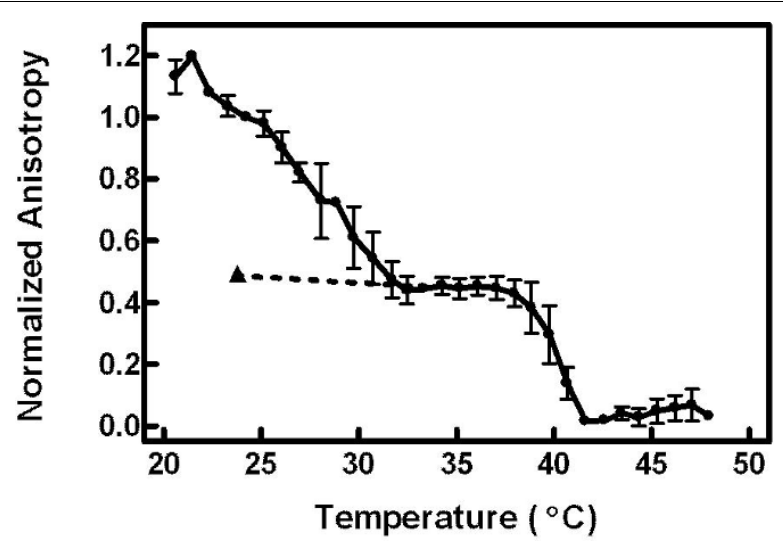

Figure 2 Detailed anisotropy temperature profile. Anisotropy was measured at $585 \mathrm{~nm}$ in single degree increments from 20 to $48^{\circ} \mathrm{C}$. Following the final measurement at $48^{\circ} \mathrm{C}$, the sample was cooled to $25^{\circ} \mathrm{C}$ and anisotropy was re-assessed (triangle, dotted line). The data were normalized to the anisotropy value at $25^{\circ} \mathrm{C}$ (set to 1.0 ) and to the minimum value obtain in the profile (set to 0.0 ) prior to aggregation of results. Error bars represent the SEM for three vesicle samples. 


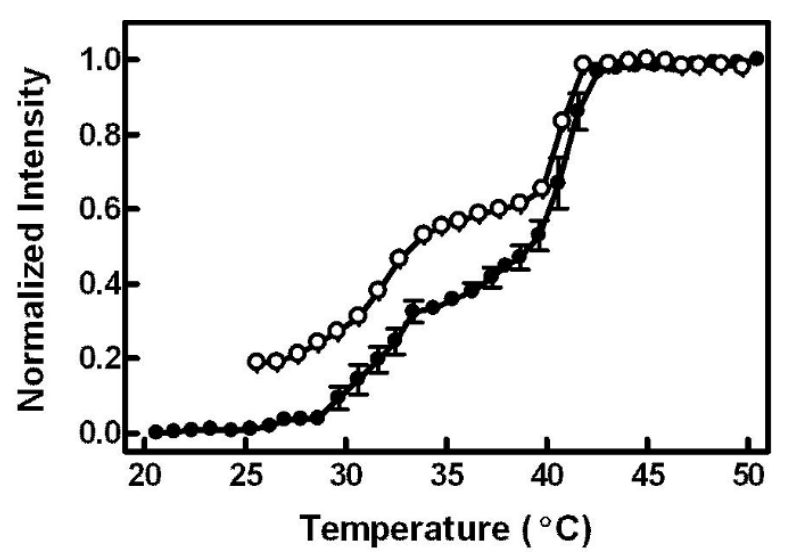

Figure 3 Detailed emission intensity temperature profile. Emission intensity (at $585 \mathrm{~nm}$ ) was collected in single degree increments from 20 to $50^{\circ} \mathrm{C}$ for vesicles that had been stored at $4^{\circ} \mathrm{C}$ (solid circles). Error bars represent the SEM for five vesicle samples. The open circles denote a sample that was equilibrated at $50^{\circ} \mathrm{C}$ prior to cooling the sample to $25^{\circ} \mathrm{C}$ and then assaying the emission intensity at single degree intervals back up to $50^{\circ} \mathrm{C}$. Data were normalized and scaled to the maximum intensity (set to 1.0) and the intensity of refrigerated samples at $25^{\circ} \mathrm{C}$ (set to 0.0 ).

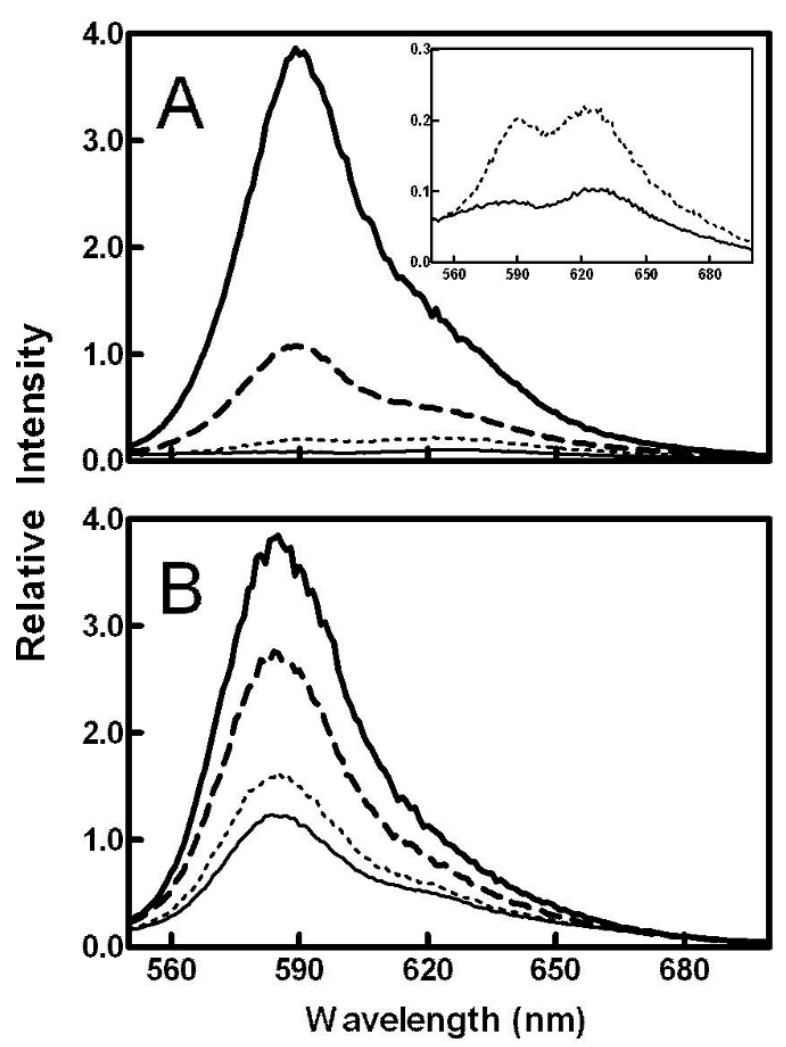

Figure 4 Emission intensity spectra for refrigerated and equilibrated vesicles. Emission spectra were collected at 25 (thin solid curve), 30 (dotted), 35 (dashed), and $45^{\circ} \mathrm{C}$ (heavy solid). Panel A: Vesicles were refrigerated for at least $24 \mathrm{~h}$ prior to fluorescence measurements. The inset displays the details of the 25 and $30^{\circ} \mathrm{C}$ spectra. Panel B: Vesicles were equilibrated at $50^{\circ} \mathrm{C}$ prior to adjusting to the experimental temperatures used in Panel A. 
peak, indicating that the membrane properties of the $\mathrm{L}_{\mathrm{c}}$ ' phase may contribute more to dimer fluorescence than those of the $\mathrm{L}_{\beta}{ }^{\prime}$ phase (Figure $4 \mathrm{~B}$ ).

Differences in the shape of the spectra were quantified by calculating the ratio of fluorescence intensity at the two spectral peaks (Figure 5). The detailed temperature profiles of the ratios from refrigerated (solid circles) and equilibrated (open circles) vesicles revealed certain similarities to the pair of intensity curves in Figure 3. At temperatures below $28^{\circ} \mathrm{C}$, a normalized ratio of 0.6 corresponding to the $\mathrm{L}_{\beta}$ ' phase was evident in the data from the equilibrated vesicles, and the influence of the $\mathrm{L}_{\mathrm{c}}$ ' phase was observed as a much lower ratio (normalized to $\sim 0.05$ ) in the refrigerated vesicles. However, unlike the emission intensity (Figure 3), the ratio of intensities remained constant as the lipids passed through the main transition, indicating no change in the shape of the emission spectra between the $\mathrm{P}_{\beta}$ ' and $\mathrm{L}_{\alpha}$ phases (Figure 5).

\section{Effect of cholesterol}

Figures 6A summarizes the combined observations of MC540 anisotropy (squares), emission intensity (open triangles), and emission peak ratio (solid inverted triangles) for equilibrated DPPC vesicles. To test the effect of the liquid-ordered $\left(L_{o}\right)$ phase, the experiments were repeated with vesicles containing various concentrations of cholesterol (up to 40\%). Cholesterol is known to abolish the pre-transition (thus eliminating the $\mathrm{P}_{\beta}{ }^{\prime}$ phase) and, at concentrations above $25 \mathrm{~mol} \%$, establish an $\mathrm{L}_{\mathrm{o}}$ phase that spans the temperature range from about 25 to $50^{\circ} \mathrm{C}$ [24-26,45]. Consequently, addition of cholesterol altered the temperature trends of anisotropy, intensity, and peak ratio (Panels B: $15 \%$ cholesterol, and C: $40 \%$ cholesterol). In general, the temperature profiles were smoother as cholesterol content increased, a result consistent with other reports of cholesterol effects on membrane properties [1,26,39,40,46]. Additionally, due to the loss of the $\mathrm{P}_{\beta}$ ' phase upon addition of cholesterol, the plateaus that existed for all three measurements between about 36 and $39^{\circ} \mathrm{C}$ were eliminated and replaced by continuous changes in properties reaching an extreme at about $42^{\circ} \mathrm{C}$ in each case. The

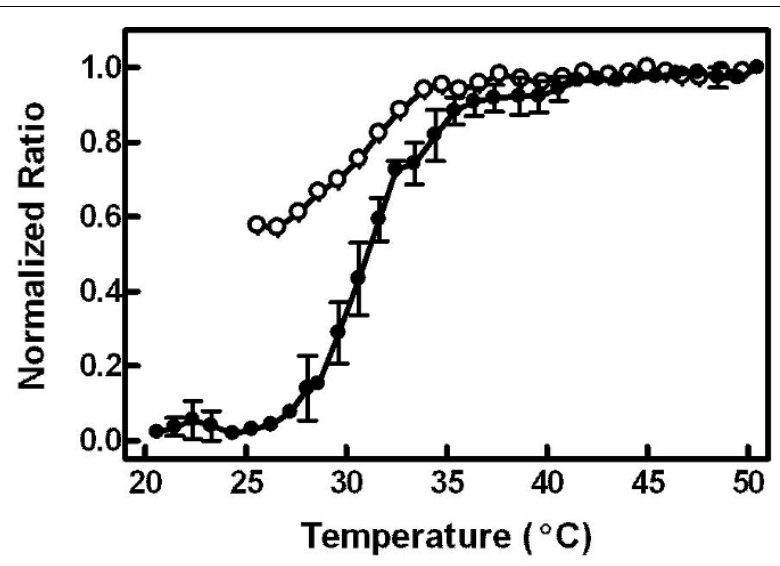

Figure 5 Detailed emission peak ratio temperature profile. Emission intensities (at 585 and $621 \mathrm{~nm}$ ) were collected in single degree increments from 20 to $50^{\circ} \mathrm{C}$ for refrigerated vesicles (solid circles), and the ratio of these intensities $(585 \mathrm{~nm} / 621 \mathrm{~nm}$ ) was calculated. Error bars represent the SEM for four vesicle samples. The open circles denote a sample that was equilibrated at $50^{\circ} \mathrm{C}$ prior to cooling the sample to $25^{\circ} \mathrm{C}$ and then assaying the emission intensity at single degree intervals back up to $50^{\circ} \mathrm{C}$. Data were normalized and scaled to the maximum ratio (set to 1.0) and the ratio of refrigerated samples at $25^{\circ} \mathrm{C}$ (set to 0.0). 

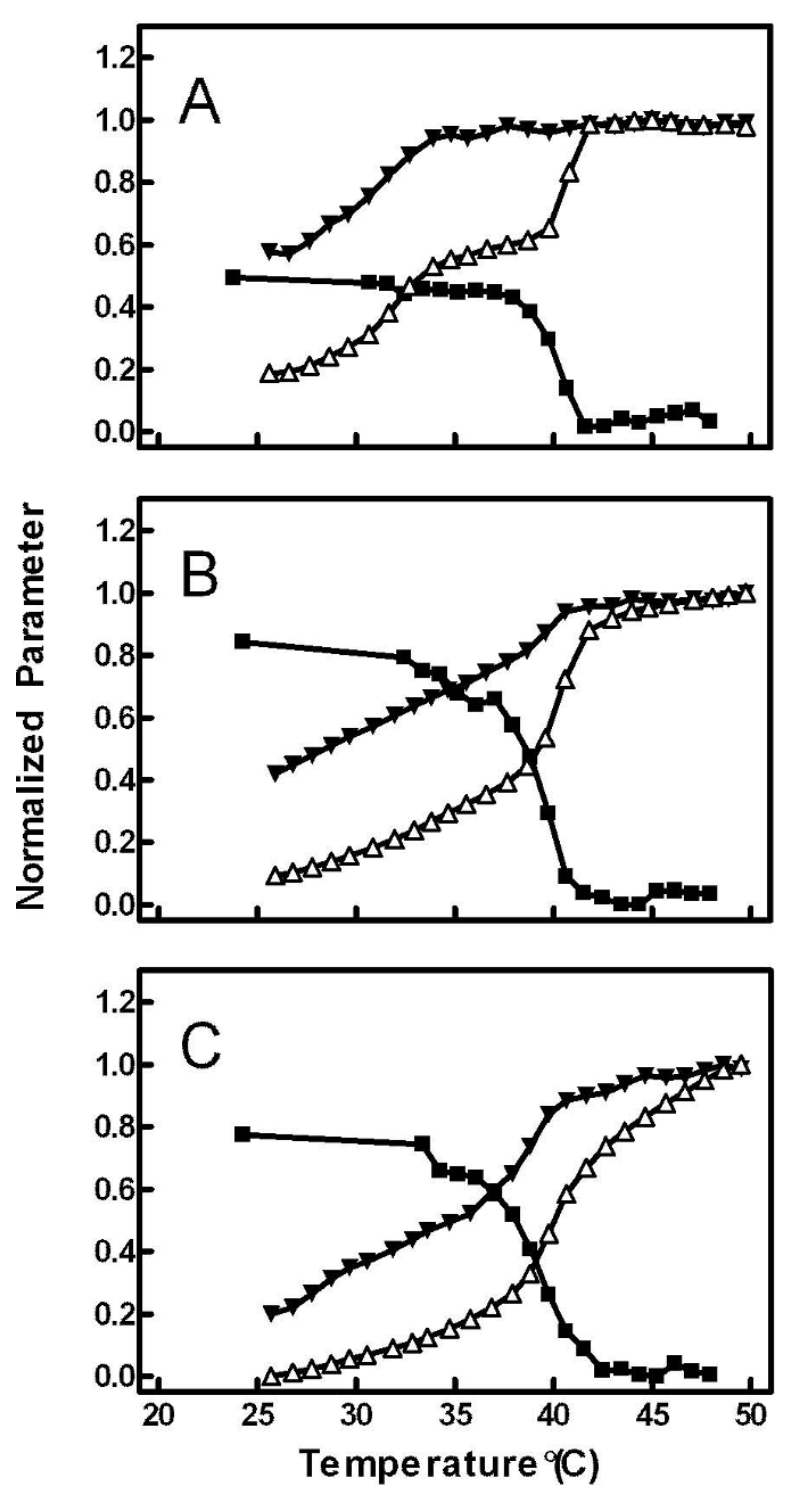

Figure 6 Effect of cholesterol. The vesicles were synthesized with 0 mol\% (Panel A), 15 mol\% (Panel B), or $40 \mathrm{~mol} \%$ (Panel C) cholesterol. The three measurements-anisotropy (solid squares), emission intensity (open triangles), and peak ratio (solid inverted triangles)-were measured for equilibrated vesicles. Data were normalized as described for Figures 2, 3, and 5.

main phase transition at $\sim 40-42^{\circ} \mathrm{C}$ present in the vesicles of pure DPPC was absent at the highest cholesterol concentrations (above 25\%) as expected (Figure 6C). Anisotropy in the $\mathrm{L}_{\beta}$ ' phase was elevated by about $60 \%$. The emission peak ratio and intensity were lower in the $\mathrm{L}_{\beta}$ ' phase compared to pure DPPC suggesting that monomer fluorescence, and therefore probably monomer binding to the membrane, is decreased by the presence of cholesterol in the solid phase.

Figure 7 summarizes data from experiments like those shown in Figure 6 as color maps of membrane physical properties detected by the various measurements of MC540 fluorescence. Changes in color brightness indicate differences in parameter values (see legend for details). By overlaying the maps, regions of differing properties relative to cholesterol content and temperature are identified. When the three 


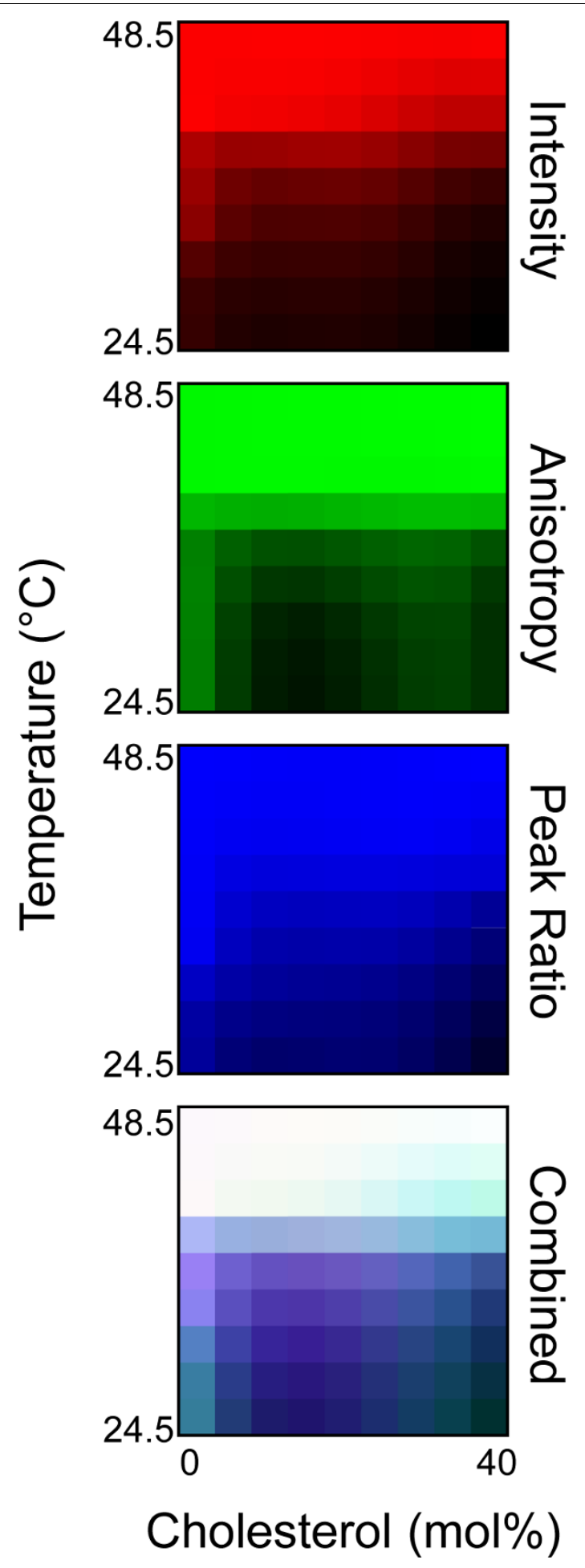

Figure 7 Temperature and cholesterol phase maps. The experiments of Figure 6 (equilibrated vesicles) were repeated at multiple cholesterol concentrations between 0 and 40 mol\%. Color phase maps were generated at temperature and cholesterol resolution of $3^{\circ} \mathrm{C}$ and $5 \mathrm{~mol} \%$ as explained in Materials and Methods. Intensity and ratio maps (red and blue, respectively) were created from the normalized values of the measurements with the brightest color representing a normalized value of 1.0. The anisotropy map (green) was created from normalized anisotropy values with the brightest color representing a normalized value of 0.0. The three maps were then overlaid to create a three-color composite map of the data (bottom panel). 
measurements co-vary, the brightness changes, but the hue remains constant. In contrast, when one or two values change more than the other(s), the hue changes toward the color of the dominant measurement $[39,40]$.

\section{Application to cultured cells}

The three steady-state measurements were repeated with living cells in the presence and absence of a drug (calcium ionophore, ionomycin) known to alter membrane properties [8]. The wavelength dependence of anisotropy was subtle, similar to that observed in Figure 1 for DPPC vesicles above the pre-transition temperature (Figure 8A). Nevertheless, both the effects of wavelength and ionomycin treatment on anisotropy were significant based on two-way analysis of variance (see legend to Figure 8 for details). No significant interaction between the two variables was observed in the analysis of variance $(p=0.99)$, suggesting that ionomycin treatment did not alter the relationship between wavelength and anisotropy. Thus, it appeared that ionomycin, while altering the membrane to allow more motion of MC540, did not change the proportion of fluorescent monomers and dimers. This interpretation was confirmed by an absence of effect of ionomycin on the peak ratio (Figure 8C). As reported
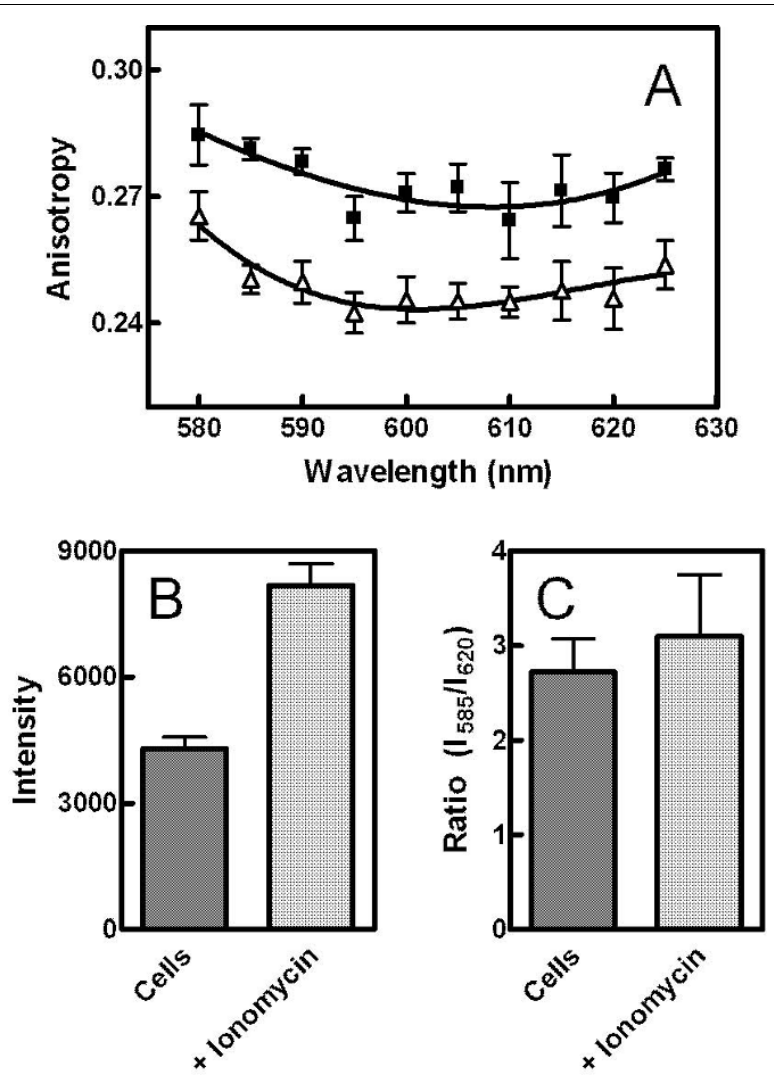

Figure 8 Application to cultured cells. Steady-state anisotropy, emission intensity, and peak ratio were assayed for $\mathbf{S} 49$ lymphoma cells both prior to and following treatment with ionomycin (300 nM). Panel A: Anisotropy was assayed at multiple wavelengths as described for Figure 1. Both the effects of ionomycin (triangles) and wavelength were statistically significant by two-way analysis of variance $(p<0.006$ in both cases, $n=9$, squares represent the untreated controls). The effect of ionomycin treatment on emission intensity at $585 \mathrm{~nm}$ was also significant (Panel $B, p<0.0001$ by paired t-test, $n=8$ ). The peak ratios (Panel C) were indistinguishable ( $p=0.33$ by paired t-test, $n=8$ ) before and after ionomycin treatment. Error bars represent the SEM. 
previously [8], a significant increase in MC540 fluorescence intensity was observed (Figure 8B).

\section{Discussion}

Evidence of all four lamellar phases was contained in the data in Figures 2, 3, and 5. Recovery from residual effects of the $\mathrm{L}_{\mathrm{c}}$ ' phase was evident as a drop in anisotropy (Figure 2) and an increase in both emission intensity (Figure 3) and peak ratio (Figure 5) at temperatures from $20^{\circ} \mathrm{C}$ up to about $32^{\circ} \mathrm{C}$ in vesicles that had been stored at $4{ }^{\circ} \mathrm{C}$. From 32 to $50^{\circ} \mathrm{C}$, the data from these refrigerated vesicles mirrored the results from vesicles that had been equilibrated by storage at room temperature or by raising temperature to $50^{\circ} \mathrm{C}$ prior to the experiment. The starting values of anisotropy, emission intensity, and peak ratio in vesicles equilibrated at $25^{\circ} \mathrm{C}$ represented the properties of the $\mathrm{L}_{\beta}$ ' phase, and the plateau in each between about 35 and $39^{\circ} \mathrm{C}$ corresponded to the $\mathrm{P}_{\beta}$ ' phase. Although the properties of the $\mathrm{L}_{\beta}$ ' and $\mathrm{P}_{\beta}$ ' phases were distinguishable by both the emission intensity and the peak ratio, measurements of anisotropy revealed identical data for both phases in equilibrated vesicles. Thus, all the changes in anisotropy in refrigerated vesicles up to $32^{\circ} \mathrm{C}$ were due to vestigial effects of the $\mathrm{L}_{\mathrm{c}}$ ' phase. The fluid phase, $\mathrm{L}_{\alpha}$, was distinguished from all other phases by high and plateaued emission intensity and minimal anisotropy values (at temperature $\geq 42^{\circ} \mathrm{C}$ ). Interestingly, the peak ratio values did not differentiate between the $\mathrm{P}_{\beta}{ }^{\prime}$ and $\mathrm{L}_{\alpha}$ phases.

As shown in Figures 6 and 7, the utility of combining three separate measurements from the same probe applies to membranes of mixed composition in addition to pure DPPC. Hence, Figure 7 illustrates resolution of multiple regions of different membrane properties as a function of both temperature and cholesterol concentration. Previous experiments studying the cholesterol phase map with MC540 concluded that the probe behaved as though it were not influenced by the presence of cholesterol [39], which was surprising based on an earlier report of a change in the MC540 emission spectrum upon addition of cholesterol [15]. While superficial inspection of any one of the three observations individually could lead to that interpretation, combining the three demonstrates clearly that the probe can resolve various regions of distinct membrane properties. In fact, these regions matched the boundaries of the traditional cholesterol phase diagram (Figure 9).

One valuable contribution of superimposing multiple measurements of MC540 fluorescence is evident as the heterogeneity of properties (color hues) in the $L_{o}$ phase. This heterogeneity has been reported previously from $\mathrm{x}$-ray diffraction studies and interpreted to represent an increase in lipid spacing as temperature is raised in the $L_{o}$ phase [47]. However, typical fluorescence observations of the $L_{o}$ phase with probes like laurdan and diphenylhexatriene do not detect any significant heterogeneity in that region of the phase diagram [39]. Hence MC540, especially in the context of multiple simultaneous measurements, is able to assess subtleties of bilayer properties at greater resolution than the more conventional steady-state methods with a single probe. An interesting question is whether the combined use of intensity, ratiometric, and anisotropy measurements will also provide insight into the superlattice structures that form at specific cholesterol concentrations [31-33]. The answer is unknown at present since increments of sterol concentration small enough to detect these structures were not used in this study. Nevertheless, since MC540 has been shown to successfully detect 


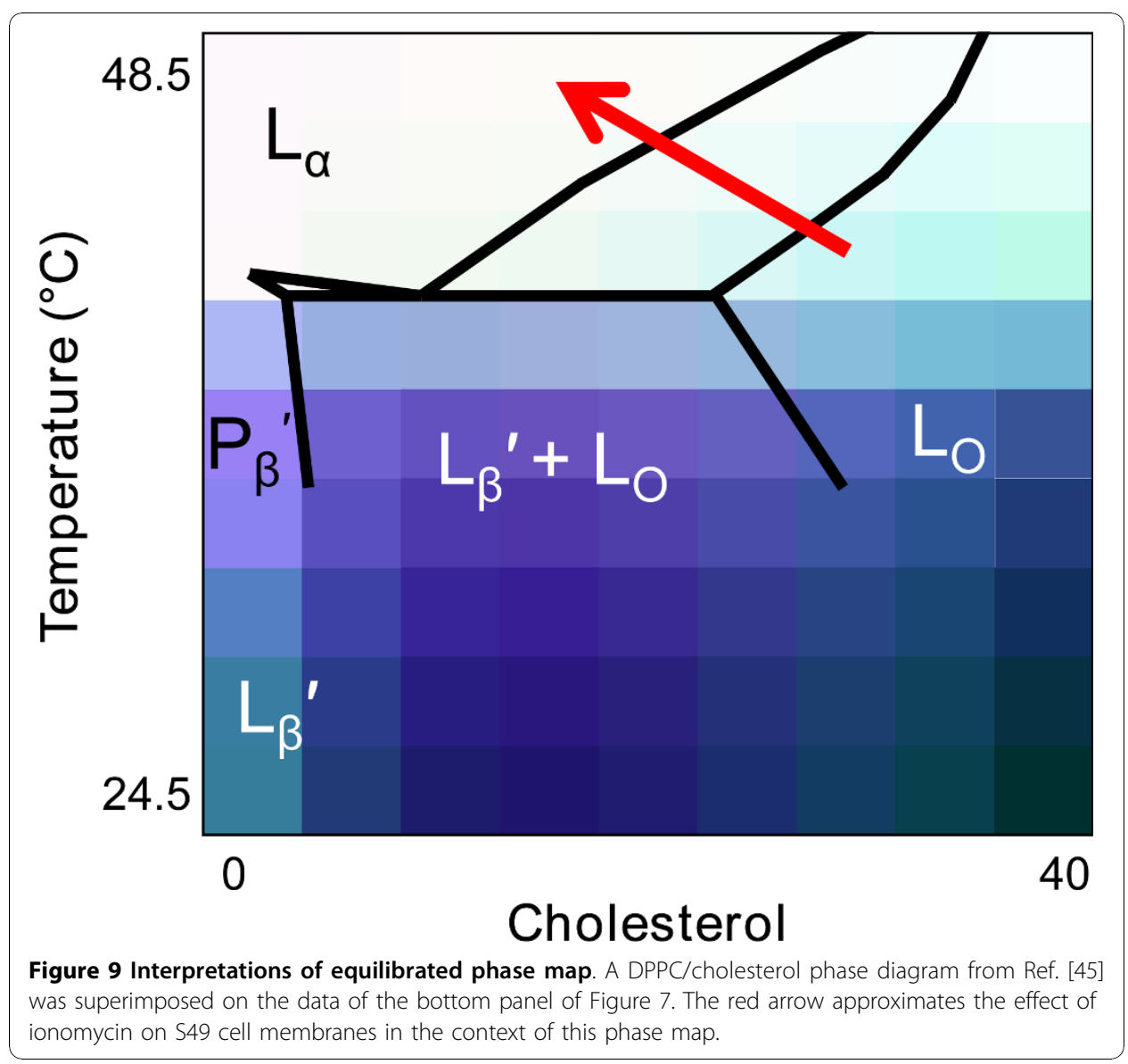

superlattice structures [33], it is likely the simultaneous measurements of multiple parameters could reveal additional information about them in future investigations.

Ordinarily, assessment of the anisotropy of fluorescent membrane probes is interpreted as an indication of membrane order and/or "fluidity." In the case of MC540, some of the temperature-dependent changes in anisotropy can also be attributed to the dynamic behavior of MC540 monomer and dimer subpopulations in the membrane. Early studies on the monomer and dimer configurations of MC540 suggested that the mobility of the dimer was greater than that of the monomer when the lipids were in the gel state [16]. These results, together with those from quenching and energy transfer experiments, were interpreted to suggest that the monomers are located near the surface of the membrane, oriented parallel to the phospholipids, and rotationally constrained due to the tight packing of the lipid heads. In contrast, the greater mobility of the dimers was attributed to a perpendicular orientation deep in the membrane [16]. The convincing wavelength dependence of anisotropy observed here at low temperatures (Figure 1) is consistent with those interpretations. Moreover, control experiments assessing differences in probe excited-state lifetimes at long and short wavelengths (as well as high and low temperature) confirmed that the changes in steady-state anisotropy observed in Figures 1 and 2 were explained mostly by differences in probe rotational rates (rather than lifetime). Although these observations and those regarding emission spectra (Figures 1, 2, and 4) corroborate the previous idea 
that MC540 dimers are fluorescent only in highly packed interfaces $[16,17,36]$, they introduce the novel finding of strong dependence on the $\mathrm{L}_{\mathrm{c}}{ }^{\prime}$ phase.

Above $32^{\circ} \mathrm{C}$, however, anisotropy values are more likely to represent the conventional interpretation of general membrane constraints on probe movement. First, the peak ratio reached a constant value at about $35^{\circ} \mathrm{C}$ (Figure 5). Inspection of the spectral data (Figure 4) confirms that this constancy is due to an absence of dimer fluorescence at these temperatures. These results suggest that dimer fluorescence vanishes at the pretransition and clarifies a previous study implying that it is eliminated by the main phase transition [17]. Second, the wavelength dependence of anisotropy observed at 15 and $25^{\circ} \mathrm{C}$ in refrigerated vesicles was nearly absent at $38^{\circ} \mathrm{C}$ (Figure 1), suggesting that spectral heterogeneity among MC540 subpopulations no longer is observed above the pre-transition temperature. Thus, additional anisotropy changes at the main transition (Figure 2) must be due to increased rotational freedom as the lipids melt.

The experiments with S49 lymphoma cells indicated that these techniques are applicable to the study of living cells. The modest but reproducible wavelength dependence of anisotropy values suggested that some of the fluorescence observed was due to MC540 dimers (Figure 8A). Although dimer fluorescence would not be expected in a fluid phase, the data of Figure $6 \mathrm{C}$ suggested a continuous trend of incremental reduction in dimer fluorescence throughout the $\mathrm{L}_{\mathrm{o}}$ phase. Since at least some of the membranes of cells are expected to have properties analogous to a $\mathrm{L}_{\mathrm{o}}$ phase (lipid rafts) [48], this result of modest wavelength dependence of anisotropy in cells makes sense. Treatment of the cells with ionomycin altered both the anisotropy and emission intensity of MC540 in the samples (Figure 8A, B). However, the wavelength dependence of anisotropy and ratio of peak intensity, i.e. both indicating relative proportion of monomer and dimer fluorescence, were not altered (Figures 8A and $8 \mathrm{C}$ ). Using the color maps created from our data, the relative changes in the membrane following ionomycin treatment were identified; the change was analogous to that observed as DPPCcholesterol vesicles transition from the $L_{o}$ to $L_{\alpha}$ phase (Figure 9, red arrow).

The data in this study revealed new insights regarding the properties of lamellar phases in DPPC vesicles and also the behavior of cell membranes during ionophore treatment. First, in addition to confirming previous reports of temperature effects on the $L_{o}$ phase, the data shown in Figure 9 suggest a much greater degree of variation across both cholesterol concentration and temperature than previously reported [39,40,47]. Second, as reviewed in [29], the molecular meaning of the mixed phase regions of the phosphatidylcholine/cholesterol phase diagram has been controversial between two disparate models, especially for the region separating the $\mathrm{L}_{\alpha}$ and $\mathrm{L}_{\mathrm{o}}$ phases: 1) a continuous transition between bordering phases without domains or 2) co-existence of domains (which may or may not necessarily represent equilibrium states) that separately reflect each of the parent phases. These two models are difficult to distinguish with experimental systems such as multilamellar vesicles that cannot be visualized directly by fluorescence microscopy, and evidence supporting both models has been recorded [29]. Interestingly, the data of Figure 9 argue that the situation is different for the transition between $\mathrm{L}_{\alpha}$ and $\mathrm{L}_{\mathrm{o}}$, which appears continuous, compared to the transition between $\mathrm{L}_{\beta}$ ' and $\mathrm{L}_{\mathrm{o}}$. In the region separating the latter two phases, the figure indicates a combination of physical properties different from either phase suggesting that co-existence of domains can create an environment unique from the 
parent phases, perhaps analogous to an alloy in metallurgy. Third, although MC540 anisotropy and spectrum peak ratio were stable as a function of temperature in the $\mathrm{P}_{\beta}$ ' phase of equilibrated vesicles (Figures 2 and 5), results from intensity measurements (Figure 3) revealed gradual changes up to the main phase transition. This observation suggests that the average spacing between phospholipids that allows binding of the probe increases continuously with temperature for DPPC bilayers in the $\mathrm{P}_{\beta}$ ' phase. Gradual changes were also observed in the $\mathrm{L}_{\beta}{ }^{\prime}$ ' phase, although some of that result probably represents the breadth of the pre-transition. Fourth, cell plasma membranes are thought to possess characteristics reminiscent of a $\mathrm{L}_{\mathrm{o}}$ phase due to the presence of cholesterol and sphingomyelin-rich domains called rafts (reviewed in [48]). The data in Figure 8 suggest that this trait shifts toward behavior more like a liquid disordered phase when the cells are loaded with calcium as illustrated in Figure 9. This outcome has significance for helping interpret studies designed to assess how the cell membrane changes during programmed cell death $[3,4,8]$.

\section{Conclusions}

In summary, the observations in this study validate our initial postulate and demonstrate the utility of multiple simultaneous steady-state measurements of MC540 fluorescence for discerning lipid phase properties with a single probe. Moreover, we have provided examples of how such measurements can provide new information regarding characteristics these phases in model and biological membranes. Previous studies with other fluorescent probes have allowed detection of more than one phase transition in membranes $[1,34,39,40]$. However, to our knowledge, this is the first example where five general lamellar phosphatidylcholine phases $\left(\mathrm{L}_{\mathrm{c}}{ }^{\prime}, \mathrm{L}_{\beta}{ }^{\prime}, \mathrm{P}_{\beta}{ }^{\prime}, \mathrm{L}_{\alpha}\right.$, and $\left.\mathrm{L}_{\mathrm{o}}\right)$ as well as multiple characteristics within those phases could be detected with a single probe using combined steady-state fluorescence measurements.

\footnotetext{
Acknowledgements

This work was supported by the National Institutes of Health (GM073997). The kind assistance of personnel (Enrico Gratton, Susana Sanchez, and Oliver Holub) at the Laboratory for Fluorescence Dynamics (University of California Irvine) is also appreciated.
}

\section{Author details}

${ }^{1}$ Department of Physiology and Developmental Biology, Brigham Young University, Provo, Utah 84602, USA..

${ }^{2}$ Department of Biology, Utah Valley State College, Orem, Utah 84058, USA..

Received: 13 July 2010 Accepted: 5 November 2010 Published: 5 November 2010

References

1. Harris FM, Best KB, Bell JD: Biochim Biophys Acta 2002, 1565:123-128.

2. Gonzalez LJ, Gibbons E, Bailey RW, Fairbourn J, Nguyen T, Smith SK, Best KB, Nelson J, Judd AM, Bell JD: PMC Biophys 2009, 2:7.

3. Laakko T, King L, Fraker P: J Immunol Methods 2002, 261:129-139.

4. Bailey RW, Nguyen T, Robertson L, Gibbons E, Nelson J, Christensen RE, Bell JP, Judd AM, Bell JD: Biophys J 2009, 96:2709-2718.

Lagerberg JW, Kallen KJ, Haest CW, VanSteveninck J, Dubbelman TM: Biochim Biophys Acta 1995, 1235:428-436. Schlegel RA, Phelps BM, Waggoner A, Terada L, Williamson P: Cell 1980, 20:321-328.

Stillwell W, Wassall SR, Dumaual AC, Ehringer WD, Browning CW, Jenski LJ: Biochim Biophys Acta 1993, 1146:136-144.

Bailey RW, Olson ED, Vu MP, Brueseke TJ, Robertson L, Christensen RE, Parker KH, Judd AM, Bell JD: Biophys J 2007, 93:2350-2362.

9. Ashman RF, Peckham D, Alhasan S, Stunz LL: Immunol Lett 1995, 48:159-166.

10. Mower DA Jr, Peckham DW, Illera VA, Fishbaugh JK, Stunz LL, Ashman RF: J Immunol 1994, 152:4832-4842.

11. Callahan MK, Williamson P, Schlegel RA: Cell Death Differ 2000, 7:645-653.

12. Fadok VA, Voelker DR, Campbell PA, Cohen JJ, Bratton DL, Henson PM: J Immunol 1992, 148:2207-2216.

13. Williamson P, Mattocks K, Schlegel RA: Biochim Biophys Acta 1983, 732:387-393.

14. Yu H, Hui SW: Biochim Biophys Acta 1992, 1107:245-254. 
15. Bernik DL, Disalvo EA: Biochim Biophys Acta 1993, 1146:169-177.

16. Verkman AS: Biochemistry 1987, 26:4050-4056.

17. Bernik D, Tymczyszyn E, Daraio ME, Negri RM: Photochemistry and Photobiology 1999, 70:40-48.

18. Chen SC, Sturtevant JM, Gaffney BJ: Proc Natl Acad Sci USA 1980, 77:5060-5063.

19. Tristram-Nagle S, Wiener MC, Yang CP, Nagle JF: Biochemistry 1987, 26:4288-4294.

20. Lentz BR, Freire E, Biltonen RL: Biochemistry 1978, 17:4475-4480.

21. Janiak MJ, Small DM, Shipley GG: Biochemistry 1976, 15:4575-4580.

22. Hong-wei S, McConnell H: Biochemistry 1975, 14:847-854.

23. Lopez MO, Freire E: Biophys Chem 1987, 27:87-96.

24. Ipsen JH, Karlstrom G, Mouritsen OG, Wennerstrom H, Zuckermann MJ: Biochim Biophys Acta 1987, 905:162-172.

25. Huang TH, Lee CW, Das Gupta SK, Blume A, Griffin RG: Biochemistry 1993, 32:13277-13287.

26. Vist MR, Davis JH: Biochemistry 1990, 29:451-464.

27. Chiang YW, Shimoyama Y, Feigenson GW, Freed JH: Biophys J 2004, 87:2483-2496.

28. Feigenson GW: Biochim Biophys Acta 2009, 1788:47-52.

29. Veatch SL, Keller SL: Biochim Biophys Acta 2005, 1746:172-185.

30. Veatch SL, Keller SL: Biophys J 2003, 85:3074-3083.

31. Chong PL: Proc Natl Acad Sci USA 1994, 91:10069-10073.

32. Liu F, Sugar IP, Chong PL: Biophys J 1997, 72:2243-2254.

33. Virtanen JA, Ruonala M, Vauhkonen M, Somerharju P: Biochemistry 1995, 34:11568-11581.

34. Chang HH, Bhagat RK, Tran R, Dea P: J Phys Chem B 2006, 110:22192-22196.

35. Yeagle PL: The Structure of Biological Membranes. CRC Press, Boca Raton, FL; 1992.

36. Kozhinova EA, Tikhomirova AM, Kozyr LA, Kyagova AA, Potapenko AY: Russian Journal of Physical Chemistry A 2007, 81:1335-1340

37. Bernik DL, Disalvo EA: Chem Phys Lipids 1996, 82:111-123.

38. Osada K, Kodama T, Yamada K, Sugano M: Journal of Agricultural and Food Chemistry 1993, 41:1198-1202.

39. Wilson-Ashworth HA, Bahm Q, Erickson J, Shinkle A, Vu MP, Woodbury D, Bell JD: Biophys J 2006, 91:4091-4101.

40. Stott BM, Vu MP, McLemore CO, Lund MS, Gibbons E, Brueseke TJ, Wilson-Ashworth HA, Bell JD: J Lipid Res 2008 , 49:1202-1215.

41. Wilson HA, Huang W, Waldrip JB, Judd AM, Vernon LP, Bell JD: Biochim Biophys Acta 1997, 1349:142-156.

42. Lewis RN, McElhaney RN: Biophys J 1992, 61:63-77.

43. Lewis RN, Mak N, McElhaney RN: Biochemistry 1987, 26:6118-6126.

44. Langner M, Hui SW: Biochim Biophys Acta 1993, 1149:175-179.

45. Ipsen JH, Mouritsen OG, Zuckermann MJ: Biophys J 1989, 56:661-667.

46. Mannock DA, Lewis RN, McElhaney RN: Biophys J 2006, 91:3327-3340.

47. Clarke JA, Heron AJ, Seddon JM, Law RV: Biophys J 2006, 90:2383-2393.

48. Barenholz Y: Subcell Biochem 2004, 37:167-215.

doi:10.1186/1757-5036-3-14

Cite this article as: Franchino et al:: Combined use of steady-state fluorescence emission and anisotropy of merocyanine 540 to distinguish crystalline, gel, ripple, and liquid crystalline phases in

dipalmitoylphosphatidylcholine bilayers. PMC Biophysics 2010 3:14. 